\title{
Detection of stimulus change: The hypothetical roles of visual transient responses
}

\author{
LEW B. STELMACH, CHARLES M. BOURASSA, and VINCENT DI LOLLO \\ University of Alberta, Edmonton, Alberta, Canada
}

\begin{abstract}
Two random-dot patterns (D1 and D2) were displayed briefly in close temporal succession, separated by an interstimulus interval (ISI). The two patterns were identical except that D2 could contain one more element (addition) or one less element (subtraction) than D1. Observers were required to detect the addition or subtraction using a two-alternative forced-choice method. Three experiments were designed to examine Phillips and Singer's (1974) suggestion that detection of additions and subtractions can be explained in terms of neural ON and OFF responses. Experiment 1 replicated Phillips and Singer's finding that performance is progressively impaired as the ISI is increased. In Experiment 2, sudden shifts in luminance between D1 and D2 were combined with several durations of ISI to produce transient responses in a novel manner. Experiment 3 explored the roles of transient neural responses and of configurational changes as determinants of performance. Most predictions based on Phillips and Singer's model were confirmed by the experimental outcomes. Some discrepancies between theory and data are noted, and directions for further research are suggested.
\end{abstract}

Sudden changes in stimulus luminance produce transient bursts of neural (ON or OFF) activity in the visual system. Phillips and Singer (1974) note that, in everyday experience, these changes in luminance occur when vision is temporarily obstructed, as in the case of an object seen through moving branches. Under these circumstances, it is advantageous not only to maintain a continuous perception of the object but also to detect changes such as appearance or disappearance of features while the visual field is temporarily obscured. Observers' ability to detect the appearance or disappearance of a feature under conditions in which the visibility of the scene is interrupted, was studied by Phillips and Singer.

In Phillips and Singer's (1974) experiments, observers were required to detect a focal event that was either an appearance (addition) or disappearance (subtraction) of one stimulus element (small square) in a display that contained, on average, 50 identical elements. Each trial consisted of two successive displays separated by a variable interstimulus interval (ISI), ranging from 20 to $500 \mathrm{msec}$. The first display (D1) contained a random configuration of stimulus elements, which remained on view for $500 \mathrm{msec}$. The second display (D2), also shown for $500 \mathrm{msec}$, was either the same as D1 or differed in one of two ways: D2 could contain one more element (addition) or one less element (subtraction) than D1. Observers used the "yes-no" method to indicate whether D2 differed

\footnotetext{
This research was supported by Grant $\mathrm{A} 6592$ to the third author. We wish to thank Margolee M. Horn for experimental and clerical assistance. Requests for reprints should be sent to Lew B. Stelmach, Department of Psychology, University of Alberta, Edmonton, Alberta, Canada T6G 2E9.
}

from D1. Detection of the focal element became progressively impaired as the ISI between D1 and D2 was increased. Overall, additions were easier to detect than subtractions. The results were explained in terms of a neurophysiological model, which postulated significant levels of inhibitory interactions between $O N$ and $O F F$ responses.

Broadly speaking, Phillips and Singer's (1974) proposals are consistent with other physiological hypotheses of how ON and OFF responses interact within the visual system. For example, Schiller (1982) reported evidence that $\mathrm{ON}$ and $\mathrm{OFF}$ responses in primates are processed within channels that are independent peripherally but become interactive at cortical levels. Further physiological evidence consistent with this hypothesis has been provided by Levine and Shefner (1977).

Psychophysical evidence pertinent to this issue has been obtained almost exclusively at threshold levels of stimulation (e.g., Krauskopf, 1980; Rashbass, 1970; Roufs, 1974). The outcomes of these studies were not uniform: some (e.g., Rashbass, 1970) concluded that $\mathrm{ON}$ and OFF transients were processed along a single channel; others (e.g., Krauskopf, 1980) found evidence for two separate channels. But inferences drawn from studies with liminal stimuli may not describe accurately the functioning of $\mathrm{ON}$ and OFF responses at levels well above threshold. Indeed, at suprathreshold levels, everyday experience argues compellingly for two channels, witness our ability to distinguish increments from decrements in the luminance of comfortably visible objects. But the question remains: are ON and OFF channels entirely independent at suprathreshold levels, or do they interact, as Phillips and Singer (1974) have suggested? 
Aside from the seminal work of Phillips and Singer, there is a notable dearth of evidence bearing directly on this question. The experimental paradigm employed by Phillips and Singer seems, at first, well suited to the psychophysical study of ON and OFF responses at suprathreshold levels. But, on close inspection, the paradigm has some shortcomings and limitations that must be rectified to permit unambiguous assessment of $\mathrm{ON}$ and OFF responses and their interactions.

The more salient shortcomings are detailed in the introduction to Experiment 1. They concern primarily response criterion biases that might have been produced by the "yes-no" method and by intermingling additions and subtractions within the same set of trials. The major limitations, examined in Experiments 2 and 3 along with other issues, concern the restrictions imposed by the paradigm on the way in which ON and OFF events can be combined at any one moment in time. To anticipate the argument detailed in the discussion of Experiment 1, Phillips and Singer's paradigm permitted simultaneous combinations of ON-focal with ON-contextual responses or of OFF-focal with OFF-contextual responses. It did not permit simultaneous combinations of ON-focal with OFF-contextual responses or vice versa. To provide a complete account of ON and OFF responses and their interactions, it is necessary to examine all four possible combinations of ON and OFF responses in focal and contextual stimuli. A novel paradigm that permits such combinations is described in Experiments 2 and 3.

In brief, the present research has two major aims. The first is to devise alternative methodologies that would obviate the possible criterion-bias problems outlined above and elaborated in Experiment 1. The second is to develop a novel paradigm capable of examining all four possible combinations of $O N$ and OFF responses. In addition, the novel paradigm was combined with that of Phillips and Singer in Experiments 2 and 3 to provide converging evidence bearing on Phillips and Singer's proposals.

\section{EXPERIMENT 1}

Examination of Phillips and Singer's paradigm reveals two methodological problems, both of which involve response biases. The first is related to the "yes-no" method. In order to respond, the observer must decide whether a focal event occurred on a particular trial. Given a conservative criterion and some degree of uncertainty, the observer would tend to respond "no." This would produce fewer correct detections, but also fewer false alarms. Alternatively, a liberal criterion would produce more "yes" responses and would result in more correct detections, but also in more false alarms. In either case, sensory effects and response criterion effects would be confounded.

Changes in criterion level were probably responsible for some differences in Phillips and Singer's results under experimental conditions in which identical results should have been expected. For example, in Phillips and Singer's (1974) Figure 4B (D1 = $2 \mathrm{msec}$, D2 $=500 \mathrm{msec}$, ISI = $100 \mathrm{msec}$ ), the focal event was detected with approximately $50 \%$ accuracy, yet in their Figure 5 (D1 =2 $\mathrm{msec}, \mathrm{D} 2=500 \mathrm{msec}$ ), the interpolated detection accuracy at $\mathrm{ISI}=100 \mathrm{msec}$ was approximately $70 \%$. False-alarm rates in the two conditions covaried with detection accuracy, strongly suggesting that fluctuations in observers' response criteria were responsible for these differences.

Another source of unwanted variance in Phillips and Singer's original paradigm might have been the mixture of additions and subtractions within one set of trials. Observers could, for example, adopt a strategy favoring the detection of additions over subtractions, thus maximizing their performance on additions to the detriment of subtractions. Informal trials in our laboratory showed that observers could easily concentrate on one type of focal event while attending less to the other. It is possible that this type of response strategy may have produced the observed differences between additions and subtractions reported by Phillips and Singer (1974).

Experiment 1 attempted to deal with the methodological concerns outlined above. Fluctuations in observers' response criteria were minimized by replacing the "yes-no" method with a two-alternative temporal forced-choice method known to be virtually criterion-free (Scharf, 1975). On each trial, two D1D2 sequences were displayed. The focal event (either an addition or subtraction) occurred unpredictably in the first or in the second sequence; the observer's task was to identify the sequence containing the focal event.

Unwanted variance that could arise from unequal allocation of attention to additions or subtractions was reduced by blocking trials, so that a set of trials contained only additions or only subtractions. In all other respects, the temporal parameters of Experiment 1 replicated the corresponding experiment in Phillips and Singer's (1974) report.

\footnotetext{
Method

Observers. The first and third authors and one other subject served in the experiment. All subjects had normal or correctedto-normal vision and were experienced with the experimental task.

Visual display. Each display consisted of a random configuration of 41 elements presented within a notional square $(1.5 \times$ $1.5 \mathrm{~cm}$ ) on a Hewlett-Packard 1333A oscilloscopic point plotter equipped with fast P15 phosphor. A photograph of a typical display is shown in Figure 1. As can be seen, each display element was composed of five closely packed dots. At a viewing distance of $28 \mathrm{~cm}$, which was set by a headrest, each dot subtended a visual angle of approximately $0.08 \mathrm{deg}$, and the separation between ad-
} 


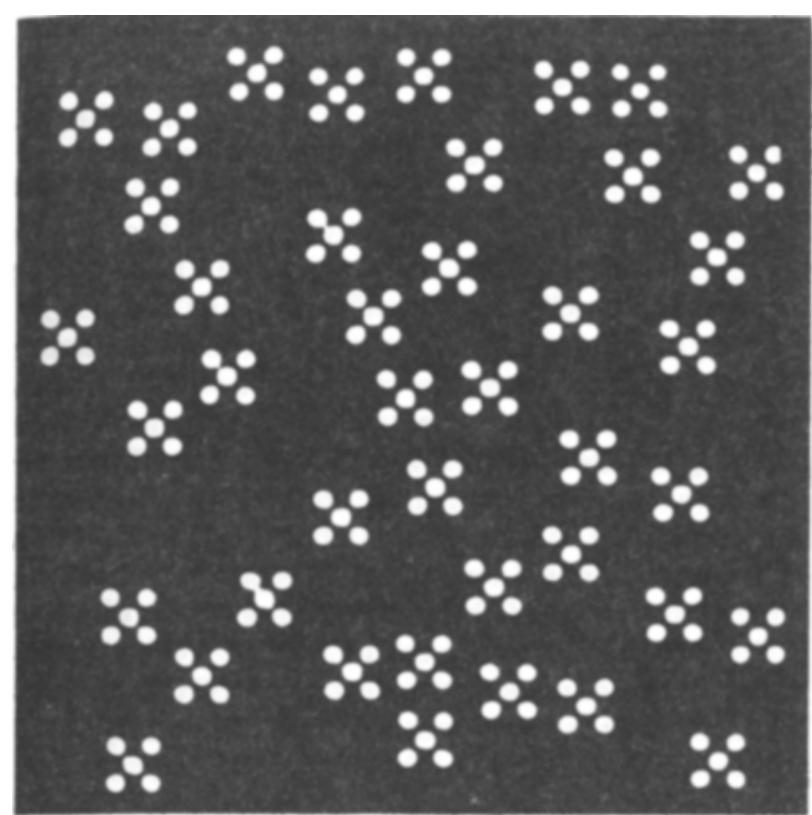

Figure 1. Typical display consisting of $\mathbf{4 1}$ elements.

jacent corner dots within an element was also approximately $0.08 \mathrm{deg}$. Each display element subtended a visual angle of approximately $0.24 \mathrm{deg}$, and the display as a whole subtended a visual angle of approximately $3.0 \mathrm{deg}$. The minimal separation between elements was approximately $0.2 \mathrm{deg}$.

The oscilloscope's luminous intensity was kept constant from day to day with a calibration procedure similar to that described by $\mathrm{Di}$ Lollo (1979). In essence, the procedure involved plotting a test patch in the center of the display surface and measuring its luminance with a Tektronix J16 digital photometer. The gain of the oscilloscope was adjusted regularly so that the test patch produced a constant reading on the photometer.

Design and Procedure. Observers sat facing the oscilloscope's screen in a mat-white booth illuminated by an overhead flourescent light source. Background luminance was maintained at a constant value of $31 \mathrm{~lx}$ measured by the Tektronix J16 photometer at the blank surface of the oscilloscope's screen. Display luminance was set to a comfortable viewing level. The test patch plotted at this level produced a reading of $35 \mathrm{~lx}$ on the $\mathrm{J} 16$ photometer. The lux scale was used in preference to the more appropriate nits scale $\left(\mathrm{cd} / \mathrm{m}^{2}\right)$ because the illuminance probe of the $\mathrm{J} 16$ photometer permitted greater resolution.

The display was shown for two successive 500 -msec periods, referred to as D1 and D2, respectively, separated by interstimulus intervals (ISIs) of $0,10,20,40,80,160$, or $320 \mathrm{msec}$. On each trial, two D1-D2 pairs were displayed, with the focal event occurring randomly in either the first or the second pair. There were two types of focal events: D2 could be displayed either with one element less than D1 (subtraction) or with one element more than D1 (addition). Within any given session, the type of focal event (addition or subtraction) was held constant. The configuration of elements was randomized separately for each D1-D2 pair.

In detail, the sequence of events on each trial was as follows: four fixation dots defined a square area subtending approximately $4.5 \mathrm{deg}$ of visual angle. Upon a button press by the observer, the fixation dots disappeared, and the first D1-D2 sequence was displayed. The fixation dots then reappeared, and the observer pressed the button again to display the second D1-D2 sequence. The observer then responded by depressing one of two buttons to indicate whether the focal event had occurred in the first or in the second sequence, as required by the two-alternative temporal forced-choice method.
All scoring, timing, and display functions were performed by a PDP-8/L computer. Each observer served for two sessions in each of the 14 experimental conditions defined by the factorial combination of ISI ( 7 levels) and type of focal event (addition or subtraction). Each session contained 50 trials of either additions or subtractions, and lasted approximately $5 \mathrm{~min}$. ISI was constant within a session. Sessions were randomized with the restriction that each observer complete one session in each condition before beginning the second session. Thus, each observer contributed 100 observations in each of the 14 conditions.

\section{Results}

Figure 2 shows mean number of correct detections in each experimental condition, averaged across all three observers. Accuracy of performance diminished as the ISI between D1 and D2 increased. Additions were easier to detect than subtractions, although the magnitude of this difference varied with ISI. The graphical evidence for these effects was supported by the outcome of a 7 (levels of ISI) $\times 2$ (additions or subtractions) analysis of variance (ANOVA). The effect of ISI was significant $[F(6,12)=118.8, p<.01]$, as was the effect of focal event $[F(1,2)=24.57$, $p<.04]$. The interaction between ISI and focal event was also significant $[F(6,12)=4.08, p<.02]$.

\section{Discussion}

Our results are consistent with those of Phillips and Singer (1974), suggesting that any biases introduced by response criterion shifts or unequal division of attention, although noticeable in some experi-

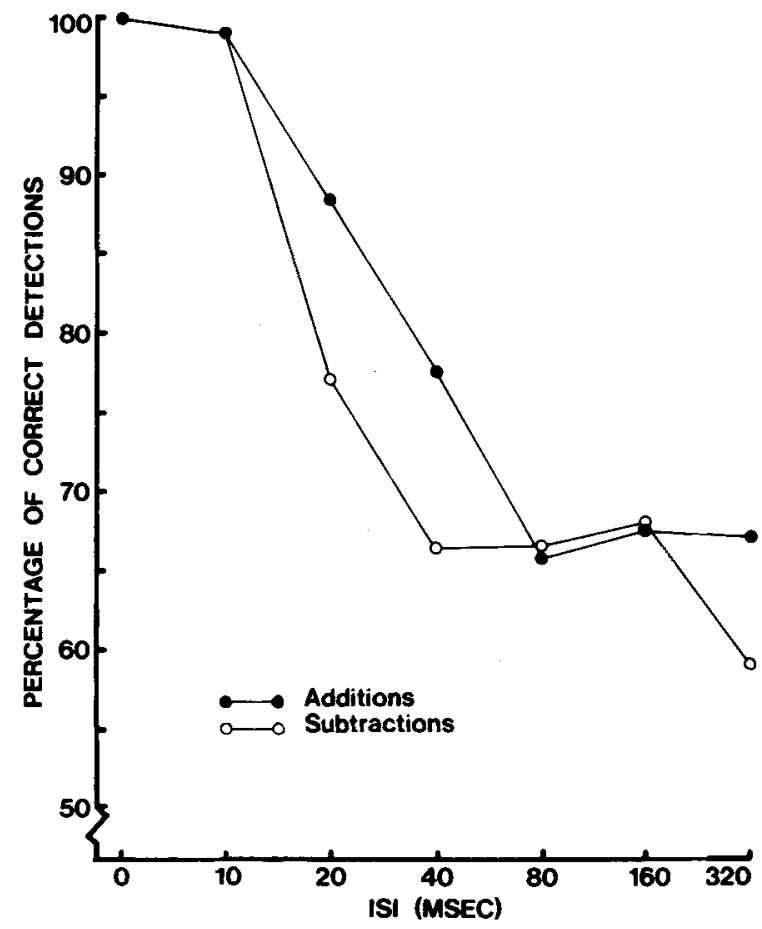

Figure 2. Mean percentage of correct detections in Experiment 1. The ISIs have been spaced logarithmically on the abscissa to avoid congestion of the points at the shorter durations. 
ments, were negligible in Experiment 1 of their report. However, overall, our results were displaced towards shorter values of ISI. This displacement may be due to a higher ambient luminance in our experiment. Higher luminance increases the detectability of brief temporal gaps in visual displays (e.g., Hecht \& Schlaer, 1936). Thus, higher ambient luminance may reduce the time constant of the visual system. Hence, the displacement towards shorter values of ISI in our results can be ascribed to a shorter time constant due to higher ambient luminance than in Phillips and Singer's study. This is also consistent with phenomenological reports of Phillips and Singer's observers who were unable to detect an ISI of $20 \mathrm{msec}$, whereas the same ISI was easily detectable in our experiment.

Phillips and Singer's explanation. Phillips and Singer accounted for the results of their experiment with a model of lateral geniculate nucleus (LGN) functioning. In essence, the model proposes that reciprocal inhibitory interactions between transient ON and OFF responses mediate performance in the experimental task. The model assumes that the onset of an element produces an ON response and that the termination of an element produces an OFF response. This is represented schematically in Figure 3. The temporal parameters of the experimental paradigm are shown at the top of the figure; immediately below are histograms of hypothetical neural firing rates of LGN cells. ON-center cells are believed to increase their firing rates in response to the onset of a display element, whereas OFF-center cells are believed to increase their firing rates in response to the termination of an element. Thus, in the section of Figure 3 labeled "contextual response," the peaks of activity in the histograms correspond to the onset of D1 and D2 in the left portion and to the termination of D1

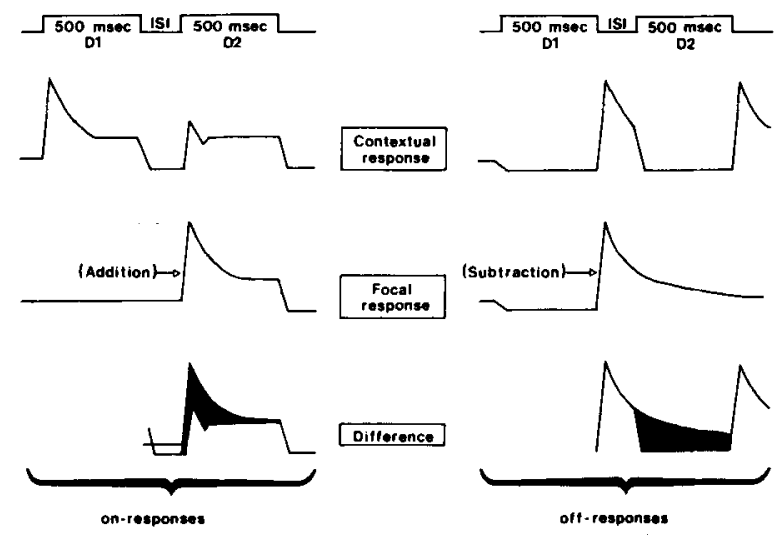

Figure 3. Temporal parameters employed in Experiment 1 are shown in the top section. Hypothetical responses to contextual and to focal stimull are shown in the middle two sections. Differences between contextual and focal responses are shown in the bottom section. ON responses are illustrated on the left and OFF responses on the right. (After Phillips \& Singer, 1974.) and D2 in the right portion. Responses of the cells are termed ON and OFF responses, respectively.

Mutually antagonistic reciprocal inhibition is an important characteristic of these cells; activity in one class tends to inhibit activity in the other. Temporal contiguity of ON and OFF activity is one factor that determines the strength of reciprocal inhibition. For example, if an ON response closely trails an OFF response, or vice versa, the magnitude of both responses will be attenuated relative to the case where the two are separated by a longer interval. In the present paradigm, ON and OFF responses occurred in close temporal contiguity when D1 and D2 were separated by a short ISI. This explains why, in the left section of Figure 3 labeled "contextual response," the peak in the histogram of the ON response at the onset of D2 is smaller than the peak at the onset of D1. Similarly, it explains why the histogram of the OFF response to the termination of D1 shows an abrupt decline at the point where $\mathrm{D} 2$ begins.

Note that the interactions are local. Thus, the OFF response produced by an element at the termination of D1 inhibits and is inhibited by the ON response produced by an element at the onset of D2 only if both elements are in close retinal alignment. This is true for all elements that are displayed both in D1 and D2 (termed “contextual elements"). The section labeled "contextual response" in Figure 3 portrays the neural responses produced by any one of the contextual elements.

Focal elements differ from contextual elements because they are displayed in only one period, either in D1 (subtraction) or in D2 (addition). As a result, the neural response to the focal element occurs in relative temporal isolation; it neither precedes nor follows another response in the same retinal location. Consequently, the neural response to the focal element is uninhibited, and for this reason it is stronger than the contextual responses. The section of Figure 3 labeled "focal response" shows histograms of hypothetical neural responses to an addition and to a subtraction. Each focal response is stronger than the corresponding contextual ON or OFF response. This, in turn, explains why the focal response is discriminable from the contextual responses. To illustrate this more clearly, the histograms of focal and contextual responses have been superimposed in the section of Figure 3 labeled "Difference." The amount by which the focal response exceeds the contextual response is shaded in. The greater amplitude of focal responses can be ascribed, in the case of additions, to the absence of a preceding OFF response, and, in the case of subtractions, to the absence of a trailing $O N$ response. Phenomenologically, additions were reported by one observer as appearing brighter than the contextual elements. Another observer perceived subtractions as dimmer. The other observers were uncertain. 
Magnitude of reciprocal inhibition between $\mathrm{ON}$ and OFF responses depends critically on their temporal contiguity. As duration of ISI increases, so does the temporal separation between the two types of responses, with consequent reduction in the magnitude of inhibitory interactions. Undiminished by inhibitory effects, ON and OFF responses produced by contextual stimuli become indistinguishable from those produced by focal stimuli. This is why, at the longer ISIs, observers are relatively poor at detecting the occurrence of focal events.

Further empirical considerations. On the whole, the outcome of Experiment 1 is in agreement with Phillips and Singer's hypothesis. The range of ISIs over which performance is seen to deteriorate most rapidly (0-80 msec; Figure 2$)$ is congruent with estimates of the duration of brief neural events such as ON and OFF responses (Winters \& Walters, 1970). Phillips and Singer's hypothesis can also account for the advantage of additions over subtractions within the 0-80-msec range of ISIs (see Figure 2, and the significant interaction effect between ISI and type of focal event). According to the hypothesis, additions were easier to detect than subtractions because the magnitude of the difference between focal and contextual responses was greater for ON responses than for OFF responses. This can be seen in Figure 3 in which the difference between OFF responses is restricted to the tail of the response, whereas the difference between $\mathrm{ON}$ responses includes the entire response.

It must be noted, however, that the relatively higher detectability of ON responses is not a universal finding. Breitmeyer and Julesz (1975) found that ON responses were more easily detected only at low spatial frequencies; at frequencies higher than about 5 cycles/ deg ON and OFF responses were of approximately equal detectability. On the other hand, using incremental and decremental contrast thresholds, Patel and Jones (1968) found OFF responses to be more easily detectable. Level of background luminance may be a factor in these contrasting results. Short (1966) found that OFF responses were more detectable than ON responses, but only at low levels of background luminance. At higher levels, $O N$ and OFF responses were equally detectable. On the basis of these somewhat complex results, Phillips and Singer's (1974) account of the relatively higher detectability of ON responses, though plausible, remains open to question.

At ISIs beyond about $80 \mathrm{msec}$, the performance curves for additions and subtractions converged to approximately the same level (Figure 2). That the curves should converge to a common level is not surprising because, at ISIs beyond about $100 \mathrm{msec}$, the effect of transient neural events should be negligible (Singer \& Phillips, 1974). What is surprising, however, is that the curves converge well above the $50 \%$ level that would be expected on the basis of chance alone. Clearly, at the longer ISIs, observers were able to detect differences between D1 and D2 on the basis of some other type of processing activity whose duration exceeds that typically associated with sensory events. Experiment 3 examines the possibility that the basis for such discrimination may consist of configurational changes between D1 and D2 brought about by the addition or subtraction of the focal element. Before addressing this issue, however, we describe a new paradigm which implements all four possible combinations of ON and OFF focal and ON and OFF contextual stimuli. In this respect, the new paradigm permits a more comprehensive investigation of any interactions that may take place between $\mathrm{ON}$ and OFF visual channels. It can also be used in combination with the original paradigm to examine further the notions proposed by Phillips and Singer.

A novel paradigm. In Phillips and Singer's (1974) original paradigm, $\mathrm{ON}$ and OFF responses were generated by an ISI. Consequently, a focal OFF response could occur at the end of D1 along with all other OFF responses produced by the contextual elements. Similarly, a focal ON response could be produced only at the onset of D2, along with other ON responses produced by the reappearing contextual elements. In more general terms, it was not possible to produce focal ON responses simultaneously with contextual OFF responses, nor was it possible to produce simultaneous OFF-focal and ON-contextual responses. This is a limitation of the paradigm because $O N$ and OFF focal events could not be studied in the context of neural responses of the opposite sign occurring simultaneously. At a more theoretical level, this meant that possible interactions between ON and OFF visual channels could not be studied directly.

These limitations were circumvented in the new paradigm by producing transient $\mathrm{ON}$ and OFF responses independently of ISI. The alternate way of producing transient responses was suggested by De Valois, Jacobs, and Jones's (1962) finding that a sudden increment or decrement in luminance of a steady display produces ON and OFF responses, respectively, whose strength is proportional to the magnitude of the stimulus change. This method was implemented in Experiment 2 by introducing a discrete shift in luminance (increment or decrement) simultaneously with the occurrence of the focal event (addition or subtraction).

As well as being more comprehensive, this method offers an additional advantage in that it provides an alternative means of examining the basis of Phillips and Singer's (1974) proposals. That is, in view of the central role played by $O N$ and OFF responses in Phillips and Singer's model, an alternative method for producing such responses would be clearly valuable. Consonant results obtained with the two para- 
digms would provide converging evidence for the determining role of such transient responses.

\section{EXPERIMENT 2}

According to Phillips and Singer (1974), the target element (say, an addition) is distinguishable from the remaining elements to the degree to which the strength of its ON response exceeds that of the contextual elements. In the limiting case, when the ISI is zero, an addition is detected easily because no contextual ON responses occur at the same time. If the distinctiveness of additions is to be reduced, an ISI of nonzero value is necessary.

As an alternative to employing nonzero values of ISI, D2 could be displayed at an intensity level significantly higher than that of D1. The sudden increment in intensity of the contextual elements at the transition from D1 to D2 would produce contextual ON responses (De Valois et al., 1962) which would reduce the distinctiveness of the addition. In this paradigm, the sudden increment in intensity would be functionally equivalent to the reappearance of the contextual elements after an ISI. By the same token, the two paradigms provide different-although equally appropriate-means of evaluating Phillips and Singer's (1974) proposals. Indeed, the paradigms can be employed in a complementary fashion to verify specific theoretical predictions. Namely, shifts in luminance may be combined with variations in ISI to modulate the strength of the inhibitory interactions hypothesized by Phillips and Singer.

For example, suppose we employ an ISI of $40 \mathrm{msec}$ to separate a dim D1 from an appreciably brighter D2. The higher luminance of D2 would act in conjunction with the onset of the reappearing elements to counteract the inhibitory effects of the immediately preceding contextual OFF responses. The net result would be to increase the similarity between the neural responses to the uninhibited focal element and to the neighboring contextual elements. In turn, it may be expected that additions would be more difficult to detect under these conditions than when D1 and $\mathrm{D} 2$ are displayed at the same level of luminance. In Experiment 2, two levels of luminance were combined factorially with seven durations of ISI to examine the joint effects of ISI and of luminance shifts on the detectability of additions and subtractions.

\section{Method}

With the exceptions noted below, observers, displays, design, and procedures were the same as in Experiment 1. The salient modifications pertained to the luminance of D1 and D2. Four luminance combinations were employed. In Condition LL, both D1 and D2 were plotted at a low level of luminance; in Condition HH, both were plotted at a high level; in Condition LH, D1 was plotted at the low level and D2 at the high level; lastly, in Condition HL, D1 was plotted at the high level and D2 at the low level. The low level of luminance $(L)$ was defined as the level at which the intensity test patch, mentioned earlier, produced a read- ing of $35 \mathrm{~lx}$ on the Tektronix J16 photometer. Similarly, the high luminance level produced a reading of $312 \mathrm{~lx}$. In all other respects, Experiment 2 was identical to Experiment 1.

Specific treatment conditions in Experiment 2 were defined by the factorial combination of seven durations of ISI $(0,10,20$, $40,80,160$, and $320 \mathrm{msec}$ ), two types of focal events (addition or subtraction), and four combinations of luminance (LL, HH, LH, HL). Data for the LL condition were already available from Experiment 1 and were not duplicated in Experiment 2. The 42 new conditions in Experiment 2 were sequenced in the same fashion as in Experiment 1 .

\section{Results}

Figure 4 shows the mean number of correct detections in each experimental condition, averaged across all three observers. The data for Condition $\mathrm{LL}$ were those of Experiment 1. The individual data were analyzed by a 2 (additions vs. subtractions) $\times 4$ (luminance combinations) $\times 7$ (ISI) ANOVA. All main effects and interactions were significant at least at the .002 level. Clearly, the results of this analysis must be interpreted in light of the significant interaction effect among all three factors $[F(18,36)=3.85$, $p<$ $.0004]$.

\section{Discussion}

Conditions LL and HH. Despite appreciable differences in absolute levels of luminance, Conditions LL and HH yielded virtually identical results (Figure 4). This outcome is entirely consistent with expectations based on Phillips and Singer's (1974) model. In terms of the model, absolute level of luminance has little or no effect on detectability of the focal event, provided that D1 and D2 have the same luminance. This is so because detectability of the focal event depends not on the absolute strength of the neural responses produced by focal and contextual stimuli but on their relative strengths. From the diagrams illustrated in Figure 3, it can be seen that the relative strengths of focal and contextual responses, as determined by inhibitory interactions, would be unaffected by changes in the absolute heights of the curves within each diagram. To wit, in Condition $\mathrm{HH}$ strong $\mathrm{ON}$ responses would be counterweighed by strong preceding OFF responses, whereas

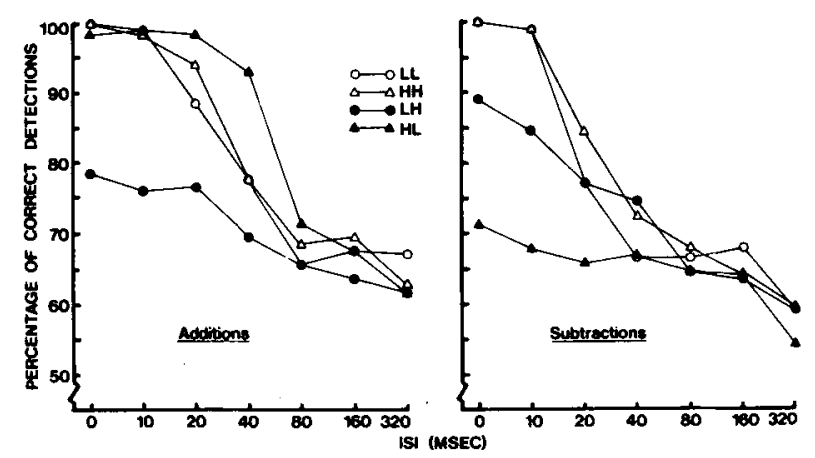

Figure 4. Mean percentage of correct detections in Experiment 2. (Note logarithmic spacing on the abscissa.) 
in Condition LL weak ON responses would be counterweighed by weak OFF responses.

It should be stressed that this remarkable invariance of the visual system has notable ecological significance in that it permits unimpaired visual functioning over a broad range of ambient luminances.

Conditions LH and HL. In the left and in the right panels of Figure 4, the relative levels of the $\mathrm{LH}$ and the HL curves are reversed. Namely, in the left panel (additions), $\mathrm{HL}$ is above $\mathrm{LH}$, whereas in the right panel (subtractions), HL is below LH. It may be noted that the reversal is most prominent at ISIs shorter than $80 \mathrm{msec}$.

At a coarse level of analysis, this pattern of results is consistent with expectations based on Phillips and Singer's model. For example, consider the relationship between the $\mathrm{LH}$ and $\mathrm{HL}$ curves for additions, shown in the left panel of Figure 4 . In the case of LH, detection of the added element was severely impaired by the concurrent increment in luminance of the contextual elements. Phenomenologically, confusability between the added focal element and the contextual elements was greater in this condition than in any of the remaining three conditions.

According to theory, discriminability of the added focal element depends on the degree to which the strength of its neural ON response exceeds that of the contextual ON responses. Now, compare Condition LH with either control condition (LL or $\mathrm{HH}$ ) at an ISI of zero. Since, in the control condition, there was neither an ISI nor a luminance shift, the ON response produced by the focal element occurred in relative isolation. In the $\mathrm{LH}$ condition, on the other hand, the focal ON response was accompanied by a volley of other $\mathrm{ON}$ responses produced by the sudden brightening of the contextual elements (De Valois et al., 1962). As a consequence, discriminability of the focal response was enhanced in the low-noise control condition but was impaired in the relatively higher noise produced in the $\mathrm{LH}$ condition.

Performance in condition LH continued to be depressed at ISIs greater than zero. This result is congruent with theory if it is realized that the difference between focal and contextual ON responses was smaller in Condition LH than in either control condition. The greater similarity of focal and contextual responses in Condition LH can be understood as follows. First, consider the strength of the $\mathrm{ON}$ responses produced by the reappearance of the contextual elements following the ISI and ignore, for the moment, the inhibitory effect of the preceding OFF responses produced at the termination of D1. Strength of ON responses would be identical in Conditions $\mathrm{LH}$ and $\mathrm{HH}$ because the luminance of $\mathrm{D} 2$ was identical in the two conditions. But, second, in actuality, the inhibitory OFF responses produced in Condition LH would be far weaker than those produced in Condition $\mathrm{HH}$ because luminance of $\mathrm{Dl}$ was lower in $\mathrm{LH}$ than in
HH. As a consequence of weaker inhibitory interactions, contextual ON responses at the onset of D2 were stronger in Condition $\mathrm{LH}$ than in Condition $\mathrm{HH}$. Since the uninhibited focal element had the same strength in both conditions, its relative prominence over the contextual elements-and hence its detectability-was correspondingly poorer in Condition LH.

Consider now the much higher level of performance in Condition HL. According to theory, the strong OFF responses at the end of D1 acted to inhibit the strength of the ON responses of the reappearing contextual elements at the beginning of D2. Hence, the uninhibited focal element stood out more prominently-and was detected more easily-in Condition HL than in the other three conditions.

This pattern of results illustrates how ISI and luminance shifts combined in determining the level of task difficulty. In the control conditions, LL and HH, ISI was the sole source of inhibitory effects. However, ISI combined with luminance shifts as a determinant of performance in the remaining two conditions. More specifically, the sudden dimming in the HL shift moderated the effect of ISI seen in the control conditions by generating more powerful OFF responses at the end of D1 to inhibit the weak contextual ON responses produced by the dim D2. In contrast, the sudden increment in luminance in the LH shift augmented the effect of ISI seen in the control conditions by generating, at the onset of D2, powerful contextual $\mathrm{ON}$ responses which could withstand easily the inhibitory effects of the weak OFF responses produced by the dim D1.

Turning now to the results of subtractions (Figure 4, right panel), Phillips and Singer's (1974) model provides again a suitable conceptual framework. A detailed derivation of predictions will not be attempted. Suffice it to say that, just as the ON response produced by an addition was more difficult to detect in the context of other ON responses, so the OFF response produced by a subtraction was more difficult to detect in the context of other OFF responses. Reversal of the relative positions of the $\mathrm{LH}$ and $\mathrm{HL}$ curves, in the right panel as compared with the left panel of Figure 4, is to be expected if one notes that the critical factors determining detectability of subtractions are the same as for additions; only now the roles of ON and OFF responses are exactly reversed. The graphical evidence for the reversal (Figure 4) was confirmed by the significant three-way interaction mentioned above.

One discrepant aspect of the results must be noted. The reversal of the $\mathrm{LH}$ and HL curves in the two panels of Figure 4 is not quantitatively symmetrical. According to theory, subtractions in the LH condition should have yielded results identical to additions in the HL condition. Instead, contrary to theoretical expectations, the $\mathrm{LH}$ curve for subtractions was notably lower than the HL curve for additions. In a 
general sense, this can be taken to mean that detectability of focal OFF responses occurring among contextual ON responses is relatively more impaired than the detectability of focal ON responses occurring among contextual OFF responses. This effect was shown reliably in the results of each observer, and clearly merits further investigation.

\section{EXPERIMENT 3}

Experiment 3 was designed to clarify some elements of discordance between theory and data in Experiments 1 and 2. Phillips and Singer's analysis of inhibitory interactions between ON and OFF responses suggests that the degree to which the ON responses of the contextual elements reappearing in D2 are inhibited by the preceding OFF responses (or vice versa) should diminish as ISI is increased. This implies that focal and contextual responses should become progressively more similar and, therefore, that level of performance should deteriorate continuously as the duration of the ISI increases, eventually reaching chance level when transient contextual and focal responses become indistinguishable. These inferences are supported by the pronounced downward trends illustrated in Figures 2 and 4. However, two aspects of the data are at odds with these implications. First, the relationship between accuracy of performance and ISI exhibits a sharp discontinuity at ISIs of about 40-80 msec. Second, accuracy of performance is still above the $50 \%$ chance level, even at an ISI of $320 \mathrm{msec}$.

Both the discontinuities and the asymptotic levels in Figures 2 and 4 point to the possibility that more than one basis for judgment may have been employed by observers in Experiments 1 and 2. For example, detection of the focal event could be based not only on the relative strengths of focal and contextual responses, but also on changes in overall configuration of the display resulting from addition or subtraction of an element. To cite a trivial, but illustrative, example, suppose that D1 consisted of $\mathbf{4 0}$ elements arranged in four rows of 10 elements each, and that D2 contained 1 additional element at the beginning of the fifth row. Clearly, D1 and D2 could be discriminated easily on the basis of configurational differences even at very long ISIs, without the necessity of neural transient responses. In the present case, differences in relative strength may have provided a reliable basis for judgment at the shorter ISIs. But, at the longer ISIs, when differences in response amplitude become unreliable, observers may have employed changes in configuration between D1 and D2 as a basis for judgment. The sharp discontinuity in Figures 2 and 4 may identify the point at which the switch between bases of judgment occurred.

Experiment 3 was a test of this hypothesis. On the assumption that changes in configuration would be more noticeable when the displays contained fewer elements, numerosity was varied at three levels. If changes in configuration are indeed easier to detect when the number of elements is small, it should be expected that, in displays with fewer elements, changes in configuration would provide a sound basis for judgment and hence, maintain a high level of performance even at long ISIs.

\section{Method}

In essence, Experiment 3 consisted of two replications of Experiment 2. In one replication, the number of elements in the display was 11 , in the other it was 21 . In all other respects, observers, displays, method, and procedures were the same as in Experiment 2.

The 112 separate conditions in Experiment 3 were defined by the factorial combination of ISI (seven levels), type of focal event (two levels), luminance combination (four levels), and numerosity (two levels). The 112 combinations were sequenced in the same manner as in Experiment 2.

\section{Results}

Mean results, averaged over all three observers, are shown in Figures 5, 6, 7, and 8 for Conditions $L L$, $H H, L H$, and HL, respectively. To facilitate comparison, the mean results of Experiment 2 (in which the displays contained 41 elements) have been plotted along with the results of Experiment 3 in each of the four figures. Additions are shown in the left panel of each figure, and subtractions are shown in the right panels. The three curves within each panel represent performance at each of the three levels of nu-

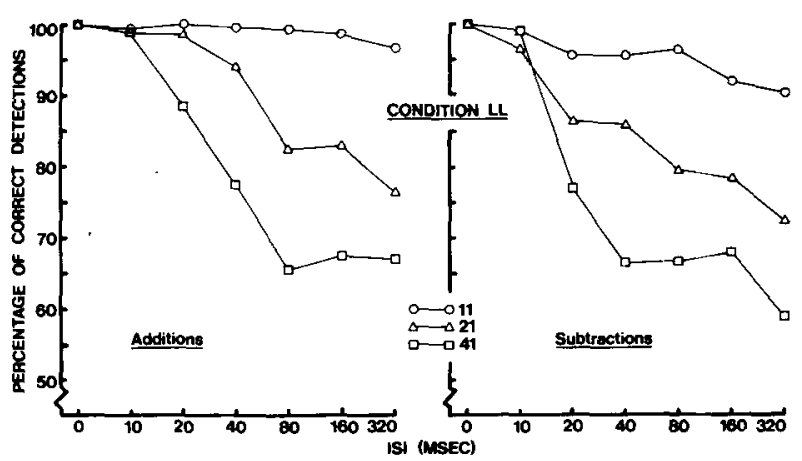

Figure 5. Mean percentage of correct detections in Condition LL of Experiment 3.

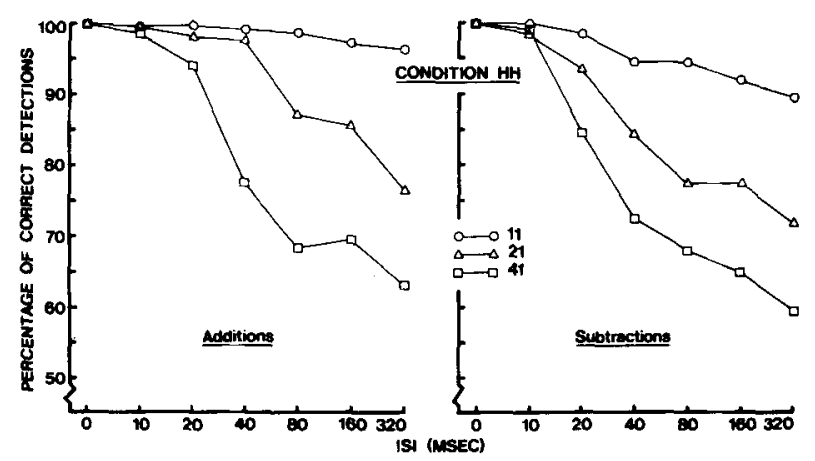

Figure 6. Mean percentage of correct detections in Condition HH of Experiment 3. 


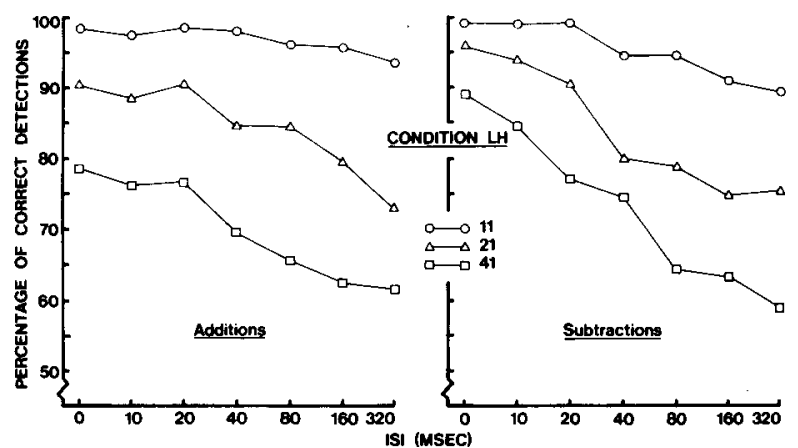

Figure 7. Mean percentage of correct detections in Condition LH of Experiment 3.

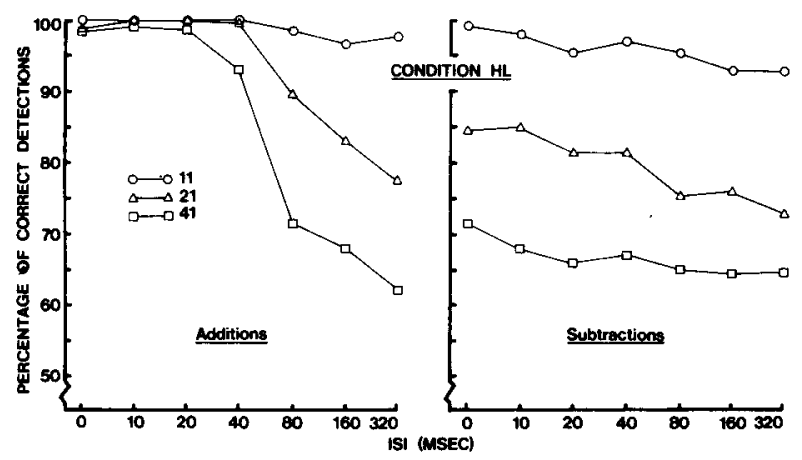

Figure 8. Mean percentage of correct detections in Condition HL of Experiment 3.

merosity. The individual data summarized in Figures $5,6,7$, and 8 were analyzed by a 2 (additions vs. subtractions) $\times 3$ (levels of numerosity) $\times 4$ (luminance combinations) $\times 7$ (ISI) ANOVA. All main effects and interactions were significant at least at the .03 level.

\section{Discussion}

All the basic findings of Experiment 2 were replicated in Experiment 3. Conditions LL and $\mathrm{HH}$ yielded virtually identical patterns of performance at each level of numerosity (Figures 5 and 6). As was the case in Experiment 2, additions and subtractions yielded qualitatively symmetrical patterns of results in Conditions LH and HL. Additions were easier to detect in Condition HL than in Condition LH, whereas subtractions were easier to detect in Condition $\mathrm{LH}$ than in Condition HL. The symmetry is clearly illustrated in Figures 7 and 8: the left panel (additions) in Figure 7 is virtually identical to the right panel (subtractions) in Figure 8. This illustrates the functional equivalence between a focal ON response in the context of other $\mathrm{ON}$ responses (LH, addition) and a focal OFF response in the context of other OFF responses (HL, subtraction). The right panel in Figure 7 and the left panel in Figure 8 are also similar, but the curves for Condition LH (subtraction) are notably lower than the corresponding curves for Condition HL (addition) at each level of numerosity. As noted in Experiment 2, this pattern of results is not consonant with the theoretical expectation of complete symmetry between the two conditions.

Effects of numerosity. The major consequence of increasing numerosity was to magnify the effects of the other variables. At the lowest level of numerosity, performance was virtually unaffected by either ISI or luminance shifts between D1 and D2. As numerosity was increased, the effects of ISI and of luminance shifts became progressively enhanced. This outcome is entirely consonant with the view that observers employed configurational changes as one of the bases for judgment in this task. When numerosity was low, configurational changes were highly discernible and were adopted as a highly reliable basis for judgment at all ISIs. By contrast, when numerosity was high, configurational changes were more difficult to detect, and observers resorted to using them as a basis for judgment only when response amplitude no longer provided a reliable means of detecting the focal event.

These considerations, coupled with the graphical evidence in Figures 5, 6, 7, and 8, permit the conclusion that the number of elements in a display (numerosity) set a floor level below which performance could not fall in any given combination of ISI and luminance shift. A corollary of this conclusion is that Phillips and Singer's paradigm cannot be used to study the role of neural transient responses in the detection of change if the number of display elements is low. This is so because, when numerosity is low, the two bases for judgment (response amplitude and configurational change) are inextricably confounded. To separate these variables, it is necessary to eliminate configurational change as a possible basis for judgment. This can be done by increasing the number of elements to a point where the floor to which performance can fall at the long ISIs is near the level of chance.

\section{GENERAL DISCUSSION}

One aim of the present work was to examine some methodological issues arising from the experiments reported by Phillips and Singer (1974). Adoption of two-alternative forced-choice methodology obviated the possible effects of response criterion biases, and displaying only one type of focal event (addition or subtraction) within a session ensured that observers did not attend selectively to one or the other type of focal event. Overall, the outcomes of the present studies were consonant with Phillips and Singer's (1974) model of detection of visual change based on inhibitory interactions between ON and OFF neural responses. 


\section{Two Complementary Paradigms}

Empirical confirmation of Phillips and Singer's (1974) notions is particularly notable in that it was obtained in the present work through two different experimental paradigms. In one paradigm, originally employed by Phillips and Singer (1974), neural ON and OFF responses were produced by means of an ISI. In the other paradigm, the neural responses were produced by sudden shifts in luminance not necessarily involving an ISI. Results obtained with the two methods were equally supportive of the presumed roles played by transient $\mathrm{ON}$ and OFF responses in the detection of change. Indeed, when the two methods were combined so that D1 and D2 were displayed at different levels of luminance and were separated by an ISI, the ON and OFF responses, supposedly produced by each method, interacted in a way that was consistent with theoretical expectations. This converging evidence from different paradigms lends notable support to the theoretical notions advanced by Phillips and Singer.

As well as providing confirmatory evidence for the role of transient responses in the detection of change, the new paradigm illustrates how more direct estimates of interactions between ON and OFF responses can be obtained. The new paradigm permits simultaneous combinations of ON-focal with OFFcontextual responses, and vice versa. In the present work, focal ON responses occurred exclusively in the context of OFF responses in Condition HL (additions) at an ISI of zero. Conversely, focal OFF responses occurred exclusively in the context of $\mathrm{ON}$ responses in Condition LH (subtractions) at zero ISI. These conditions are of particular interest because there are no a priori theoretical grounds for knowing whether detection of the focal response should be impaired equally by contextual responses of a different sign as by contextual responses of the same sign. For example, we know from Phillips and Singer's (1974) work that detectability of focal ON responses is impaired by contextual ON responses. But their work provided no evidence regarding the detectability of focal ON responses in the context of simultaneous OFF responses. The evidence provided in the present work suggests that detection of focal ON responses is far more impaired by simultaneous ON responses than by OFF responses (zero-ISI conditions in the left panels of Figures 7 and 8). Similarly, detection of focal OFF responses seems to be more impaired by simultaneous contextual OFF responses than by ON responses (zero-ISI conditions in the right panels of Figures 7 and 8 ).

This pattern of results supports the hypothesis that ON and OFF responses may be processed in separate channels. On this hypothesis, focal and contextual responses would be processed in the same channel when their signs were the same (both ON or both OFF), but would be processed in separate channels when their signs differed.
Evidence for interactions, or crosstalk, between channels may be sought in conditions in which focal and contextual stimuli were presented simultaneously but were of different signs. The combination of focal OFF responses occurring simultaneously with contextual ON responses is illustrated by the zero-ISI points in the right panel of Figure 7 (LH subtractions). It is clear that detection of the focal event (an OFF response) was impaired by the simultaneous occurrence of contextual ON responses. Furthermore, the level of impairment increased as the number of elements that produced $\mathrm{ON}$ responses was increased. This pattern of results is strongly suggestive of some degree of crosstalk between ON and OFF channels, and is consonant with Phillips and Singer's (1974) suggestion of inhibitory interactions between $O N$ and OFF responses.

The converse combination, in which focal ON responses were produced simultaneously with contextual OFF responses, is illustrated by the zero-ISI points in the left panel of Figure 8 (HL additions). In this case, detection of the focal event (an ON response) was entirely unaffected by the contextual OFF responses. Thus, an asymmetry seems to be suggested by the present results: while detection of a subtraction is impaired by contextual noise of the opposite sign, this is not true for additions. However, it would be premature to infer on this basis that crosstalk is unidirectional, namely that it occurs from ON to OFF channels but not in the reverse direction. It is conceivable, for example, that $\mathrm{ON}$ channels may be less permeable to external noise and that contextual OFF responses of greater amplitude may be required to interfere with detection of the focal ON response. The present data are insufficient to permit a conclusive statement in this regard: further parametric investigations are required in which the strengths of focal and contextual stimuli are varied systematically. Nevertheless, it is clear that the new paradigm provides a powerful means of investigating supraliminal ON and OFF responses and their interactions.

\section{Different Bases for Judgment}

Although the procedural changes implemented in the present work obviated such possible artifacts as response criterion bias, the basis on which judgments could be made was left uncontrolled. To wit, judgments could be made either on the basis of the salient transient response or on the basis of configurational change. The possibility that either or both bases of judgment could be employed in this type of task has been recognized by Phillips (1974). His suggestions regarding the relative prominence of the two judgmental bases at different durations of ISI are in close agreement with our own. Specifically, Phillips stated that "the phenomenal experience is quite different at long and short ISIs. At long ISIs, the experience is that of knowing that the two patterns are of dif- 
ferent shape, whereas at short ISIs, the experience is that of actually seeing the change happen" (Phillips, 1974 , p. 285).

In principle, it might be possible to eliminate configurational change as a basis for judgment by employing displays in which such changes are difficult or impossible to detect. In the present work, configurational changes became progressively more difficult to detect as the number of elements in the display was increased. The corresponding decrement in level of performance was ascribed to reduced availability of configurational cues as numerosity was increased. However, it is not entirely clear that the pronounced decrement in performance seen when numerosity was high (Figures $5,6,7$, and 8 ) can be ascribed solely to the progressive elimination of configurational cues. It must be realized that, as numerosity was increased, spatial separation among the elements of the display diminished correspondingly. It is possible that spatial interactions among neighboring elements might have differed significantly as a function of proximity between elements. In turn, stch spatial interactions might have affected the detectability of the focal event in different and as yet unknown ways.

Interpreting the effects of numerosity in terms of spatial interactions is rendered less plausible by data reported by Phillips (1974). Phillips varied numerosity by varying both the total area of the display and the number of elements, thus maintaining the interelement separation constant. Since the effects of varying numerosity in Phillips's (1974) experiment were similar to our own, one can infer that variations in spatial proximity, within the range explored in these studies, are not critical to the effects observed.

\section{REFERENCES}

Breitmeyer, B., \& Julesz, B. (1975). The role of ON and OFF transients in determining the psychophysical spatial frequency response. Vision Research, 15, 411-415.

De Valots, R. L., JacoBs, G. H., \& Jones, A. E. (1962). Effects of increments and decrements of light on neural discharge rate. Science, 136, 986-988.

Di Lollo, V. (1979). Luminous calibration of oscilloscopic displays. Behavior Research Methods \& Instrumentation, 11, 419-421.

Hecht, S., \& SchlaER, S. (1936). Intermittent stimulation by light: $\mathrm{V}$. The relation between intensity and critical frequency for different parts of the spectrum. Journal of General Physiology, 19, 965-979.

KRAUSKOPF, J. (1980). Discrimination and detection of changes in luminance. Vision Research, 20, 671-677.

Levine, M. W., \& SheFner, J. M. (1977). Variability in ganglion cell firing patterns; implications for separate "ON" and "OFF" processes. Vision Research, 17, 765-776.

Patel, A. S., \& Jones, R. W. (1968). Increment and decrement visual threshold. Journal of the Optical Society of America, 58, 696-699.

Phillips, W. A. (1974). On the distinction between sensory storage and short-term memory. Perception \& Psychophysics, $16,283-290$.

Phillips, W. A., \& Singer, W. (1974). Function and interaction of on and off transients in vision. I. Psychophysics. Experimental Brain Research, 19, 493-506.

RAshrass, C. (1970). The visibility of transient changes of luminance. Journal of Physiology, 210, 165-186.

Rours, J. A. J. (1974). Dynamic properties of vision - IV. Thresholds of decremental flashes, incremental flashes and doublets in relation to flicker fusion. Vision Research, 14, 831-852.

ScharF, B. (1975). Audition. In B. Scharf (Ed.), Experimental sensory psychology, Glenview, Ill: Scott, Foresman.

Schille R, P. H. (1982). Central connections of the retinal ON and OFF pathways. Nature, $297,580-583$.

Short, A. D. (1966). Decremental and incremental visual thresholds. Journal of Physiology, 185, 646-654.

Singer, W., \& Phillips, W. A. (1974). Function and interaction of on and off transients in vision. II. Neurophysiology. Experimental Brain Research, 19, 507-521.

Winte Rs, R. W., \& WALtens, J. W. (1970). Transient and steady state stimulus-response relations for cat retinal ganglion cells. Vision Research, 10, 461-477.

(Manuscript received September 26, 1983; revision accepted for publication January 6,1984 .) 